\title{
Chikungunya virus outbreak in Kerala, India, 2007: a seroprevalence study
}

\author{
Narendran Pradeep Kumar ${ }^{1 /}$, Abidha Suresh ${ }^{1}$, Perumal Vanamail ${ }^{2}$, \\ Shanmugavelu Sabesan², Kalianna Gounder Krishnamoorthy², Jacob Mathew ${ }^{3}$, \\ Varakilparambil Thomas Jose ${ }^{3}$, Purushothaman Jambulingam ${ }^{1}$
}

\author{
${ }^{1}$ Vector Control Research Centre Field Station, 5-D, Skyline River Valley Villa, Kottayam Collectorate PO, Kottayam 686002, Kerala, India \\ ${ }^{2}$ Vector Control Research Centre, Indian Council of Medical Research, Medical Complex, Indira Nagar, Puducherry, India \\ ${ }^{3}$ Rubber Research Institute of India, Kottayam, Kerala, India
}

\begin{abstract}
India was affected by a major outbreak of chikungunya fever caused by Chikungunya virus (CHIKV) during 2006-2007. Kerala was the worst affected state during 2007 with a contribution of 55.8\% suspected cases in the country. However, except for clinically reported case records, no systematic information is available on infection status of CHIKV in the region. Hence, we carried out a post-epidemic survey to estimate seroprevalence status [immunoglobulin $G(\operatorname{IgG})]$ in the community using commercially available indirect immunofluorescence test. This methodology had been reported to be highly specific and sensitive for CHIKV infection. The study area selected was the worst affected mid-highlands region of Kerala which harbour vast area of rubber plantations. The study evidenced $68 \%$ of the population to be seropositive for CHIKV IgG. Males were found more affected than females $(\chi 2=9.86$; $p=0.002)$. Among males, prevalence was significantly higher in the age classes $21-30\left(\chi_{2}=5.46 ; p=0.019\right)$ and $31-40\left(\chi_{2}=5.84 ; p=0.016\right)$ years. This may be due to high occupational risk of the male population engaged in plantation activities exposed to infective bites of Aedes albopictus. The current study provides an insight into the magnitude of CHIKV outbreak in Kerala.
\end{abstract}

Key words: Chikungunya virus - serosurveillance - India - rubber plantation - Kerala - Aedes albopictus

Chikungunya fever is caused by an arthropod-borne Alphavirus, belonging to family Togaviridae, primarily transmitted to humans through the bite of infected Aedes mosquitoes (Pialoux et al. 2007). The rapid geographic spread of the virus led to a large chikungunya disease epidemic in India during 2006-2007 (Mavalankar et al. 2007). During the 2007 Chikungunya virus (CHIKV) outbreak in India, the worst affected state was Kerala, with $55.8 \%$ of the suspected chikungunya fever cases in the country, compared with only $5.8 \%$ cases during 2006 (Kumar et al. 2008). The Department of Health and Family Welfare of the Government of Kerala reported 24,052 cases as suspected chikungunya fever during 2007, distributed across all 14 administrative districts of the state. The state of Kerala, with an area of about $1 \%$ of the country, supports about $3 \%$ of the population $(33,387,677)$ of India, with a population density of 859 people $/ \mathrm{Km}^{2}$ (Census of India 2011). This state is a land strip lying in the extreme southern region of India bordered by Western Ghats (the mountain range along the western side of India) in the east and Arabian Sea in the west. The state enjoys tropical climate with about $3,000 \mathrm{~mm}$ rainfall annually, fed by south-west and north-east monsoons. About $28 \%$ of area of the state is forested. Topographically the state could be divided broadly into three regions, viz.,

+ Corresponding author: kumarnp@hotmail.com

Received 5 January 2011

Accepted 21 September 2011 coastal belt (12\%), mid-highlands (40\%) and Ghats section (48\%). The hilly and forested districts of Kottayam and Pathanamthitta located in the mid-high lands region of Kerala were the worst affected by CHIKV, contributing to $44.33 \%$ and $14.37 \%$ of the total cases, respectively (Kumar et al. 2008). These districts have abundant rubber plantations [109, 582 ha in Kottayam (the largest area in the state) and 61,016 ha in Pathanamthitta], which supported prolific breeding of Aedes albopictus, the main vector of chikungunya infection (Kumar et al. 2008). They breed in the rainwater that accumulates in the hemispherical containers, fitted to the trunks of rubber trees, for latex collection, in the region (Kumar et al. 2008).

Earlier studies showed that east central South African strain was responsible for the 2006 outbreak in Kerala (Kumar et al. 2007). The molecular evolutionary adaptation acquired by $\mathrm{CHIKV}$ as a result of the "A226V" mutation in the $E 1$ gene and the high population density of Ae. albopictus in the region may have contributed to the widespread and renewed chikungunya fever epidemic in Kerala during 2007 (Kumar et al. 2008). No reliable data exist on the actual prevalence of chikungunya infection in the state during this outbreak, except for the clinically suspected CHIKV case records maintained by the state health machinery, which remains a severe underestimate of the problem (Kumar et al. 2008). Hence, we carried out a systematic study to estimate the magnitude of CHIKV infection in Kerala during 2007 outbreak and the results are presented here.

The immunoglobulin $\mathrm{M}(\operatorname{IgM})$ and immunoglobulin $\mathrm{G}(\mathrm{IgG})$ antibodies against CHIKV can be detected from three to six days respectively, after the onset of clinical 
symptoms, by using enzyme linked immunosorbent assay and indirect immunofluorescence test (IIFT) (Litzba et al. 2008). Persistence of IgM antibodies was found to be varying in different studies and it did not persist normally more than three-four months period, at detectable levels (Chia et al. 2010). However, IgG antibodies are reported to be detectable in convalescence and remains so for years (Chia et al. 2010). A recent multi-country study carried out reported the better utility of $\operatorname{IgG}$ in detection of chikungunya infection compared to IgM (Niedrig et al. 2009). Knowledge about the kinetics of the serological markers of CHIKV infection has been recently reviewed (Schwartz \& Albert 2010). We used CHIKV IgG EUROIMMUN immunofluorescence diagnostic kit, the only available commercial test kit, to determine the past infection status of CHIKV in the population in the worst affected mid-highlands region of Kerala. This diagnostic kit has already been evaluated in La Reunion with 100\% specificity and $95.4 \%$ sensitivity (Litzba et al. 2008).

\section{SUBJECTS, MATERIALS AND METHODS}

Study area - The study areas selected were Chethackal $\left(09^{\circ} 24^{\prime} 28.7^{\prime \prime} \mathrm{N} 076^{\circ} 50^{\prime} 10.0^{\prime \prime} \mathrm{E}\right)$, Malankara (09 $51^{\circ} 35.1^{\prime \prime} \mathrm{N}$ 076 44'42.3” $\left.3^{\prime \prime}\right)$ and Aimcompu (09.46'34.6” N $\left.076^{\circ} 41^{\prime} 13.7^{\prime \prime} \mathrm{E}\right)$ villages located respectively in the rubber plantation belt of the districts of Pathanamthitta, Idukki and Kottayam in Kerala. These districts were the worst affected by the CHIKV outbreak in the state during 2007. The population size in Chethackal, Malankara and Aimcompu was 2,016, 1,067 and 1,451, respectively. While the former two study villages were large plantations (1 under the ownership of government organization and the other owned by a corporate agency), the latter was a village with unorganized sector of small scale (mostly below 2 ha per owner) rubber plantations. In the organized sector villages the human dwellings were restricted to two-three agglomerations (labour lines) inside plantation area, while in the unorganized sector human dwellings were more dispersed, each family living in the midst of the small scale rubber plantation they owned. The entire population in these villages is found to be natives of Kerala, except a minor proportion employed as staff at the Government sector maintained Chethackal plantation. On enquiry, it was understood that all these migrants to the state were transferred to the state prior to 2006 and were residing in the plantation belt itself with their families.

Sample size and survey method - Initially, a preliminary study to estimate the sample size of the population to be surveyed was carried out, including all the three study areas. Age and gender of the specific population in the study areas were obtained through door to door enumeration. In this study, the CHIKV seroprevalence was observed to be $80 \%, 73.3 \%$ and $46.7 \%$ in Chethackal, Malankara and Aimcompu. Therefore, it was presumed that the actual prevalence might be $\sim 50 \%$ and by allowing an error of $10 \%$ (precision $90 \%$ ), with $95 \%$ confidence the minimum required sample size for a population for about 4,600 was worked out to be 360 which was about $8 \%$ of the population. This sample size was distributed across the study areas in proportion to the population size. To survey, required number of individu- als a systematic sampling technique with random start (household) in each study area was adopted and intravenous blood samples were collected during day time from all the available individuals in the selected households during March-October 2009. Details on the previous clinical history of chikungunya fevers were also collected from the patients, simultaneously.

Blood samples were transported to the laboratory at Vector Control Research Centre, Field Station, Kottayam, the same day itself and stored at $4^{\circ} \mathrm{C}$ for further processing, using the commercially available anti-Chikungunya IgG IIFT EUROIMMUN test kits (EUROIMMUN AG, Medizinische Labordiagnostika AG, Luebeck, Germany).

Method to assess seropositivity - The protocol described by Litzba et al. (2008) was followed for the in vitro determination of human CHIKV antibodies. Subjects with an $\operatorname{IgG}$ titre $\geq 1: 100$ were classified as seropositive. The IgG positivity was detected on the basis of visualization of the fluorescent green coloration, using a fluorescent microscope (EUROIMMUN LED, Eurostar Bluelight excitation filter $488 \mathrm{~nm}$, 20X magnification). Photographs of all the samples processed were taken with the help of a digital camera attached to the fluorescent microscope. Statistical analysis was done using EPISTAT software version 16 (CDC, Atlanta, USA).

Ethics - Institutional standard guidelines were followed for the collection of blood samples after obtaining their written informed consent form for participation in the study.

\section{RESULTS}

Gender/age seropositivity - Male to female ratio in the study area was 1.02 . The number of samples collected from Chethackal, Malankara and Aimcompu were 134,123 and 124, respectively. While the minimum of $8 \%$ desired level was obtained in two of the study areas, Malankara (11.5\%) and Aimcompu (8.6\%), only $6.7 \%$ of the population could be included, in Chethackal, due to unforeseen logistical problems. Considering the population of this area $(2,016)$, the sample size could be worked out to 125 (presuming the prevalence rate of $50 \%$ obtained in the preliminary survey and allowing an error of $17 \%$ with $95 \%$ confidence interval), which yielded the minimum desired level of precision of $80 \%$. There was no significant difference in the age and gender specific distribution $(\chi 2=6.33 ; p=0.275)$ of sampled individuals. Age and gender specific distribution of population and sampling is shown in Fig. 1. It was observed that among both males and females there was a fair representation of population in all the age classes. Out of 381 samples collected, $259(68 \%)$ were found to be serologically positive for IgG. The seropositivity was detected by the appearance of bright green fluorescence of CHIKV IgG positive slides. Absence of fluorescence showed that the samples were seronegative. The seroprevalence for the study areas, viz., Chethackal, Malankara and Aimcompu, was found to be $70.9 \%, 74 \%$ and $59.7 \%$, respectively (Table).

Prevalence in males $(78 \%)$ was significantly $\left(\chi^{2}=\right.$ 9.86; $p=0.002)$ higher than that of females $(62.5 \%)$. CHIKV infection prevalence was found to be age depen- 
dent only in males $(\chi 2$ for trend in proportion $=9.549$; $\mathrm{p}=0.002)$ (Fig. 2). Comparison of age specific prevalence among males and females showed that the prevalence in males was significantly higher in the two age groups, viz., 21-30 years $(\chi 2=5.46 ; \mathrm{p}=0.019)$ and $31-40$ years $(\chi 2=5.84 ; p=0.016)$ compared to that of females.

CHIKV fever history and seropositivity - Out of the 381 individuals surveyed, 303 had history of CHIK fever (typical fever, joint pain and other symptoms) and among them $250(82.5 \%)$ were found to be IgG positive implying that the history of fever is significantly $(\chi 2=139$; $\mathrm{p}=0.000)$ associated with CHIKV infection. Among 78 asymptomatic individuals, only $10(12.8 \%)$ were found to be serologically positive. Prevalence of CHIKV fever (from history) in Chethackal, Malankara and Aimcompu areas was $80.6 \%, 83.7 \%$ and $74.2 \%$, respectively. Further, there was a significant correlation $(\mathrm{r}=0.851 ; \mathrm{p}=$ 0.032 ) between age-specific prevalence of seropositive and CHIKV fever history.

\section{DISCUSSION}

The population of districts of Pathanamthitta, Idukki and Kottayam (Census of India 2001) is 1,234,016, $1,129,221$ and 1,953,646, respectively. The CHIKV suspected fever cases reported from Kerala Health records from these districts during 2007 (cases were recorded during May-October) were only 3,456 (0.28\%), $538(0.05 \%)$ and $10,662(0.55 \%)$. The present study revealed the percentage of persons infected with CHIKV IgG to be $70.9 \%$, $74 \%$ and $59.7 \%$, respectively, in these areas. Among the fever cases, $82.5 \%$ were found serologically positive for CHIKV IgG. A similar observation (70\%) was also found in the seroprevalence survey conducted in Lamu Island, Kenya (Sergon et al. 2008). Besides, in the present study, $12.8 \%$ of individuals who had no experience of fever symptoms were serologically positive for CHIKV IgG as observed elsewhere also (Sergon et al. 2007).

About 3.6 million fever cases were recorded during 2007 in Kerala $(11.3 \%$ of the total population of the state), compared with about 1.8 million in 2006 and 1.2 million in 2005. There was an upsurge of fever cases and also suspected chikungunya fever cases in 2007. It may be presumed that the actual figure on account of chikun-

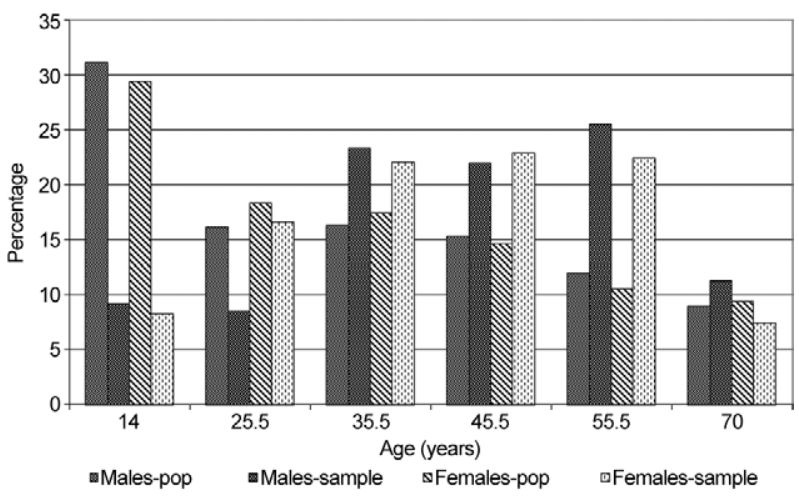

Fig. 1: age and gender distribution of the population (pop) and sampled individuals.

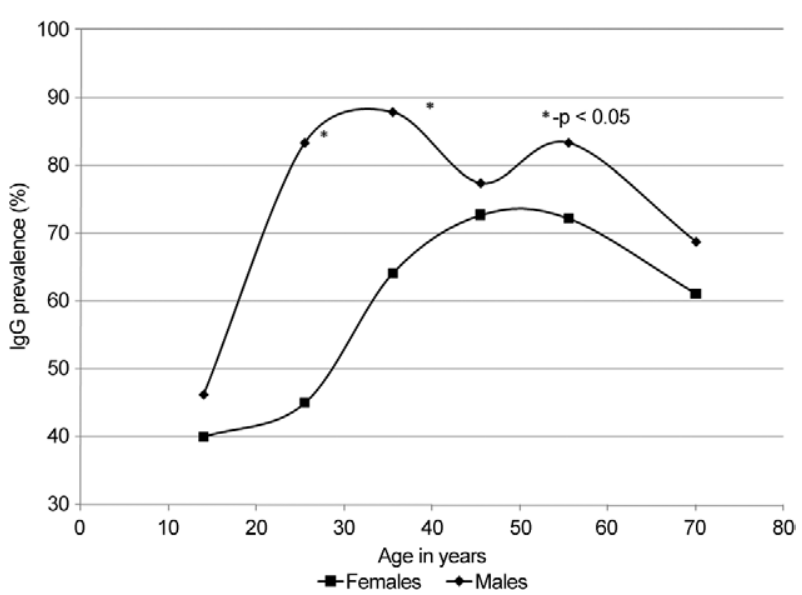

Fig. 2: age and gender specific immunoglobulin $\mathrm{G}(\mathrm{IgG})$ prevalence of chikungunya positive cases.

gunya cases would have been very high, since all fever cases had been recorded as viral fever and this might include more of chikungunya fever cases than the figures officially reported (Kumar et al. 2008). The flow of patients to the hospitals exceeded the inpatient capacity of the hospitals in the districts of Kottayam, Patha-

TABLE

Seroprevalence of study areas

\begin{tabular}{|c|c|c|c|c|c|c|c|c|c|c|}
\hline \multirow[b]{2}{*}{ Area } & \multirow{2}{*}{$\begin{array}{l}\text { Population } \\
\text { (n) }\end{array}$} & \multicolumn{3}{|c|}{$\begin{array}{l}\text { Blood samples collected } \\
\text { (n) }\end{array}$} & \multicolumn{3}{|c|}{$\begin{array}{l}\text { IIFT positives } \\
\text { (n) }\end{array}$} & \multicolumn{3}{|c|}{$\begin{array}{l}\text { Prevalence } \\
\quad(\%)\end{array}$} \\
\hline & & Male & Female & Total & Male & Female & Total & Male & Female & Total \\
\hline Chethackal & 2,016 & 57 & 77 & 134 & 45 & 50 & 95 & 78.95 & 71.43 & 70.89 \\
\hline Malankara & 1,067 & 55 & 68 & 123 & 45 & 46 & 91 & 81.81 & 67.65 & 73.98 \\
\hline Aimcompu & 1,451 & 29 & 95 & 124 & 20 & 54 & 74 & 68.97 & 56.84 & 59.68 \\
\hline Total & 4,534 & 141 & 240 & 381 & 110 & 150 & 260 & 78.01 & 62.50 & 68.24 \\
\hline
\end{tabular}

IIFT: indirect immunofluorescence testing (immunoglobulin G). 
namthitta and Idukki. Under these unprecedented circumstances, immunological diagnosis was not possible with the available facilities in the local hospitals.

The present study provides an insight on the magnitude of the CHIKV outbreak in the plantation sector of Kerala during 2007 and the figures actually projected could be an underestimate of the problem, as reported elsewhere. The seroprevalence of chikungunya infection recorded in this study was much higher $(68 \%)$ compared to the similar CHIK outbreaks in many other parts of the world. The seroprevalence rate in Italy was $10.2 \%$ in 2007, 38.2\% in the Reunion Island outbreak of 2006 and $37.2 \%$ in Mayotte (Gerardin et al. 2008, Sissoko et al. 2008, Moro et al. 2010). The higher seroprevalence rate reported in Kerala could be attributed to the exposure of the population dwelling and engaged in the rubber plantations to the infective bites of the day biting and exophilic vector species, Ae. albopictus, abundant in this region. Ae. albopictus was found to be the predominant species in the region (61.99\%). They breed profusely in the rainwater collections of the unused/discarded latex collection containers and in leaf axils of pineapple plants cultivated as inter crops in immature rubber plantations (Vector Control Research Centre/Indian Council of Medical Research Annual Report, unpublished observations). According to State Government Health authorities, the 2007 outbreak of chikungunya fever lasted for about six months. The mosquito control activities carried out were inefficient, owing to the vastness of area of the outbreak (the area of 3 districts included in the study is $9,203 \mathrm{Km}^{2}$ ) and unplanned operational measures carried out without an efficient entomological surveillance mechanism and due to unpreparedness of the health authorities to handle such a widespread outbreak. Besides, as described, rubber plantation belt in Kerala pose a special ecological and topographical situation, poorly accessible to Government Health authorities for routine surveillance due to the hilly and forested terrain, compared to urban situations. Also, about $20 \%$ of the total rubber plantation belt which are large area plantations (ranging from 2-3,000 ha) is under the jurisdiction of corporate agencies, without a systematic surveillance system from State Health Government authorities. These factors could have contributed to the higher prevalence rate of chikungunya outbreak recorded in the rubber plantation belt in Kerala.

The higher seroprevalence rate amongst males (78\%) evidenced the occupational risk of this gender of the population, who were mainly engaged as plantation workers towards rubber tapping during early morning hours (06:00 am-11:00 am) for collection of latex. Interestingly, this activity coincided with the peak hours (sunrise) of biting activity of Ae. albopictus, as recorded in the similar ecological situation (the rubber plantations) in Thailand (Thammapalo et al. 2009). Also, male workers (74\%) involved in rubber plantation related activities were significantly $(\chi 2=65.9 ; p=0.0001)$ higher than the females $(26 \%)$ as rubber tapping is a skilled job involving manual labour. Female population engaged in plantation work was mainly limited to weeding out activities in the plantations (after 09:00 am) and occasionally collection of latex milk after the tapping activity was carried out by the male members (after 11:00 am). From these plantation practices, it could be ascertained that male population engaged in rubber tapping activity during early morning hours would have been exposed to more Ae. albopictus biting activity compared to the female population. Similar observations on gender differences in seroprevalence had been recorded elsewhere which was attributed to exposure to infection due to community specific habits, customs or behaviours (Sissoko et al. 2008). Amongst the male population, the age group 21-40 years who were engaged in plantation work was found to be in the highest risk category. This observation is similar to the seroprevalence study conducted in Mayotte, where a higher seroprevalence rate $(>73 \%)$ existed in the age group higher than 25 years (Sissoko et al. 2008). Also, there are reports indicating that CHIKV IgG was higher in individuals above 15 years old, as diversity of socioeconomic status and living in makeshift housing conditions increased the risks of exposure to infection (Sergon et al. 2007, 2008, Sissoko et al. 2008). Recent studies conducted in Italy also showed that the prevalence of infection increased with age (Moro et al. 2010). They viewed that age and gender are proxy-factors for specific behaviour that causes higher exposure to Ae. albopictus bites, like staying outdoors during daytime without using any personal protection measures. Studies also showed that seroprevalence was greater in the part of the village with more human activity. Besides, differences in attack rates were likely caused by local factors, including population immunity and genetic susceptibility, vector competence, environmental settings and effectiveness of outbreak control measures (Ayu et al. 2010). In Mayotte, CHIKV seroprevalence was higher in households with structural risk factors for mosquito breeding or exposure, such as open yards (Raude \& Setbon 2009).

There is no documentation on outbreaks or cases of CHIKV infection in Kerala before 2006. Thus, the population in the state being immunologically naïve, the recent CHIKV outbreak caused tremendous anguish in the rubber plantation belt of the state, which was also highly receptive owing to the abundant vector population prevalent in the region. The present study exposes the magnitude of CHIKV infection in rubber plantation belt of Kerala and provides basic parameters for modelling the transmission potential of outbreaks and planning control measures for this arbo-viral infection in a tropical setting. Such type of studies using appropriate and reliable diagnostic measures is essential for the quantification and monitoring of epidemics, which enable development and implementation of cost effective public health measures to manage the disease outbreak situations in tropical, developing countries.

\section{ACKNOWLEDGEMENTS}

To Mr. PM Ajithlal, Mrs. Jessu Mathew and Ms. Kochurani George, VCRC Field Station, Kottayam, for technical assistance rendered for the study.

\section{REFERENCES}

Ayu SM, Lai LR, Chan YF, Hatim A, Hairi NN, Ayob A, Sam IC 2010. Seroprevalence survey of Chikungunya virus in Bagan Panchor, Malaysia. Am J Trop Med Hyg 83: 1245-1248. 
Census of India 2001. [homepage on the Internet]. Available from: censusindia.gov.in/Tables_Published/Basic_Data_Sheet.aspx.

Census of India 2011. [homepage on the Internet]. Provisional population totals. Available from: censusindia.gov.in/2011-prov-results/ census2011_PPT_paper1.html.

Chia PY, Ng MMl, Chu JJH 2010. Chikungunya fever: a review of a reemerging mosquito-borne infectious disease and the current status. Current Research, Technology and Education Topics in Applied Microbiology and Microbial Biotechnology Formatex: 597-606.

Gerardin P, Perrau J, Fianu A, Favier F 2008. Determinants of Chikungunya virus infection in the Reunion Island: results of the SEROCHIK seroprevalence survey in the population, August-October 2006. Bull Epidemiol Hebd 38: 39-40.

Kumar NP, Joseph R, Kamaraj T, Jambulingam P 2008. A226V mutation in virus during the 2007 Chikungunya outbreak in Kerala, India. J Gen Virol 89: 1945-1948.

Kumar NP, Madhu Mitha M, Krishnamoorthy N, Kamaraj T, Joseph R, Jambulingam P 2007. Genotyping of virus involved in the Chikungunya outbreak 2006 in South India (Kerala and Pondicherry). Curr Sci 93: 1412-1416.

Litzba N, Schuffenecker I, Zeller H, Drosten C, Emmerich P, Charrel R, Kreher P, Niedrig M 2008. Evaluation of the first commercial Chikungunya virus indirect immunofluorescence test. $J$ Virol Methods 149: 175-179.

Mavalankar D, Shastri P, Raman P 2007. Chikungunya epidemic in India: a major public health disaster. Lancet Infect Dis 7: 306-307.

Moro ML, Gagliotti C, Silvi G, Angelimni R, Sambri V, Rezza G, Massimiliani E, Mattivi A, Grilli E, Finarelli AC, Spataro N,
Pierro AM, Seyler T, Macini P 2010. Chikungunya virus in northeastern Italy: a serosurvey. Am J Trop Med Hyg 82: 508-511.

Niedrig M, Zeller H, Schuffenecker I, Drosten C, Emmerich P, Rumer L, Donoso-Mantke O 2009. International diagnostic accuracy study for the serological detection of Chikungunya virus infection. Clin Microbiol Infec 15: 880-884.

Pialoux G, Gauzere BA, Jaureguiberry S, Strobe M 2007. Chikungunya, an epidemic arbovirosis. Lancet Infect Dis 7: 319-327.

Raude J, Setbon M 2009. The role of environmental and individual factors in the social epidemiology of chikungunya disease on Mayotte Island. Health Place 15: 689-699.

Schwartz O, Albert ML 2010. Biology and pathogenesis of Chikungunya virus. Nat Rev Microbiol 8: 491-500.

Sergon K, Njuguna C, Kalani R, Ofula V, Onyango C 2008. Seroprevalence of Chikungunya virus (CHIKV) infection on Lamu Island, Kenya, 2004. Am J Trop Med Hyg 78: 333-337.

Sergon K, Yahaya AA, Brown J, Bedja SA, Mlindasse M 2007. Seroprevalence of Chikungunya virus infection on Grande Comore Island, Union of the Comoros, 2005. Am J Trop Med Hyg 76: 1189-1193.

Sissoko D, Moendandze A, Malvy D, Giry C, Ezzedine K, Solet JL, Pierre V 2008. Seroprevalence and risk factors of Chikungunya virus infection in Mayotte, Indian Ocean, 2005-2006: a population-based survey. PloS ONE 3: e3066.

Thammapalo S, Wonghiranrat W, Moonmek S, Sriplong W 2009. Biting time of Aedes albopictus in the rubber plantations and the orchard, the Southernmost of Thailand. J Vector Borne Dis 6: $1-6$. 